\title{
Automated Non-Destructive Testing of Roller Bearings using GMR-Sensorarrays
}

\author{
Marc Kreutzbruck, Matthias Pelkner, Thomas Erthner, Verena Reimund \\ BAM Federal Institute for Materials Research and Testing, Unter der Eichen 87, 12205 Berlin, \\ Germany \\ Marc.Kreutzbruck@bam.de
}

\begin{abstract}
Their small size together with a remarkable field sensitivity are the most prominent features of presentday GMR sensors paving the way for various applications in automated magnetic non-destructive testing (NDT). This work presents a prototype for fast and automated magnetic testing of roller bearings. A local magnetization unit excites the magnetic field inside the bearing. As a result of a design study and the following wafer fabrication the probe was equipped with NDT-adapted GMR sensor arrays, in which 48 elements measures the field response. The detection of $40 \mu \mathrm{m}$ deep defects could be resolved with a SNR of better than $20 \mathrm{~dB}$. We also report of first reconstruction results, in which the defect depth can be determined with relatively high accuracy.
\end{abstract}

Key words: giant magneto resistance, non-destructive testing, magnetic flux leakage, sensor array

\section{Introduction}

Since its discovery in $1988[1,2]$ the giant magneto resistance (GMR) effect has been intensively investigated. This led, e.g., to a major boost in computer hard drive technology by means of smaller read heads resulting in an enhanced bit density. Generally, nowadays the GMR is of interest for many other applications concerning the determination of magnetic fields due to its resistance change of $10 \%-20 \%$ at room temperature, its remarkable field sensitivity and detection limit down to the pT-range, and high frequency range [3-5]. Also, they can be easily miniaturized and their low power consumption is a further promising feature. Even though they are still relatively costly compared to their semiconducting counterpart -the Hall sensor- the GMR continuously claims further segments in the market, such as automation and production processes, automotive, cell phones, medical application and safety inspection.

The latter includes all kind of electromagnetic testing methods to test the integrity of a component. Here, the trend is driven by growing safety requirements in which industries call for reliable non-destructive testing (NDT) methods, especially when it comes to detect small surface breaking defects in the $\mu$ m-range.

In recent years, GMR sensors have been intensively used as magnetic field sensors in magnetic flux leakage (MFL) $[6,7]$ and in eddy current (EC) testing [8-12]. Due to their main promising properties - the high field sensitivity and the high spatial resolution- also small defects can be quantitatively detected paving the way for automation of the testing process.

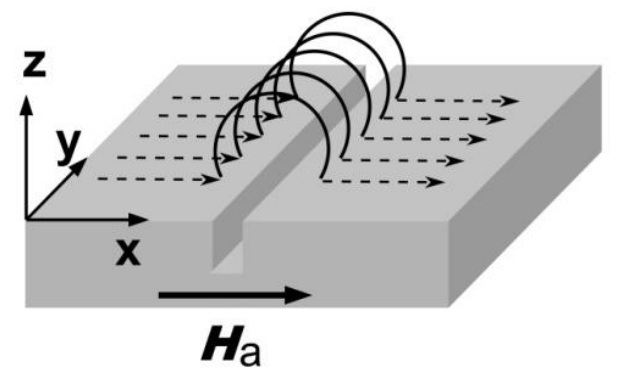

Fig. 1: Surface breaking magnetic field lines in areas of discontinuities like cracks.

In MFL-testing, a magnetized component shows a flux leakage at defect positions where the permeability significantly differs from the bulk magnetic properties (see Fig. 1). In case of conventional MFL, using magnetic particles to visualize the stray field, no quantitative estimation of the leakage field is achievable. In those cases the estimation of the crack depth turns out to be non-feasible and alternative techniques like an electrical potential probe have to be used. In contrast, the quantitative knowledge of the MFL-distribution can be obtained immediately using adapted GMR sensor arrays. In case of simple cylindrical shaped components, the GMR-based 
inspection can be automated easily and has the potential to safe inspection time compared with magnetic particle inspection (MPI). The latter includes many manual processing steps, in which besides the global magnetization step a magnetic fluid containing fluorescent magnetic particles in the sub $\mu \mathrm{m}$-range has to cover the sample under test and has to be removed again after inspection.

\section{Setup}

We developed a GMR-based automated NDTsystem for the inspection of roller bearings (see Fig. 2). This prototype (mechanical design by Astrofein $\mathrm{GmbH}$ ) is equipped with several GMR-probes to scan the whole roller bearing in less than 1 minutes. The magnetization is performed time-saving continuously by using a yoke as a local magnetization unit. The GMRsensor is placed between the two poles in the centre of the yoke. In doing so the global magnetization step and, if necessary, the corresponding demagnetization cycle can be avoided reducing the number of working steps. Also, the position of the sensor avoids the detection of background fields of the yoke itself.

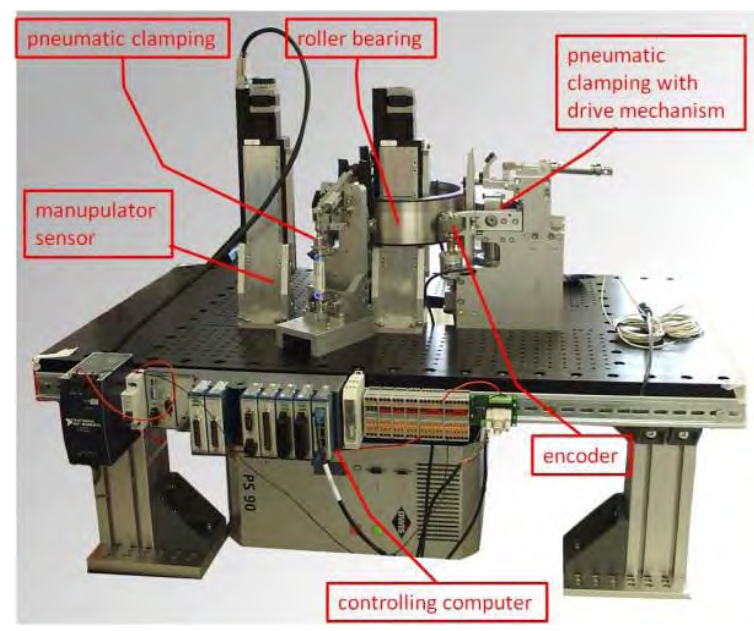

Fig. 2: Prototype for the inspection of roller bearings.

A further reduction of the inspection time can be obtained using a GMR sensor array (see Fig. 3). For this purpose we optimized a sensor array for the detection of surface breaking defects [13]. Gradiometric arranged layers were fabricated on a board with up to 48 GMR sensing elements (i.e., three 16-element arrays will be placed next to each other). The array and its complex thin film layer system was fabricated at the Sensitec $\mathrm{GmbH}$.

Usually a GMR sensor exists of four elements fabricated as a Wheatstone-bridge, resulting in a distinct reduced temperature-dependent output signal. In case of an Gradiometer layout every two elements are positioned at the same place. Both areas are divided by a certain distance (baseline). The measured quantity is the difference of the magnetic field (here the normal component with a baseline of $250 \mu \mathrm{m}$ ) between the two active areas and can be detected by measuring the bridge voltage. The size of the single element can be adjusted to the testing problem. In the following we used element sizes with a length of $190 \mu \mathrm{m}$ and a negligible height of a few $\mu \mathrm{m}$.

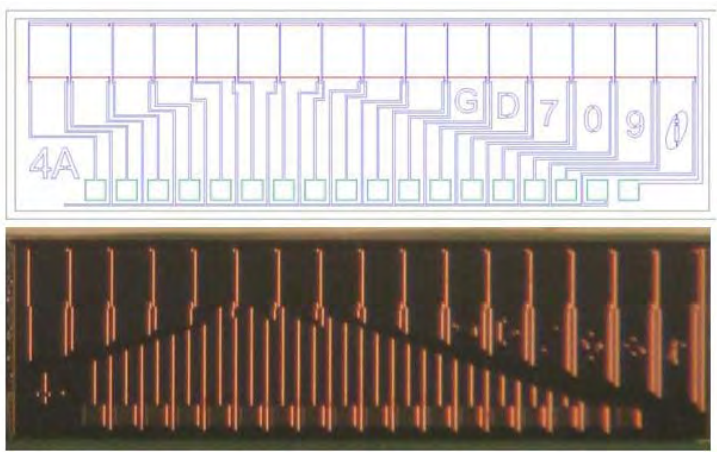

Fig. 3: GMR-array with 16 elements measuring the normal field component.

\section{Electronics}

To generate defect signals with an appropriate signal to noise ratio, an amplification of the sensor signals is required. Here, the challenge is to integrate the first amplification stage for numerous channels into a probe. Inside the probe the amount of space is limited. Therefore, the use of multiplexer is a promising way to limit the space needed for the electronics. By timesharing the amplification circuit, we were able to reduce the number of components. Also, a field programmable gate array (FPGA) is used.

In Fig. 4 a board with three 16-elements GMRArrays is presented. Every channel is red out one after another by the 16:1-multiplexer and then sent to the computer, where the data are recorded by an 18bit ADC. Onboard the signals are amplified by a factor of more than $20 \mathrm{~dB}$. The intrinsic noise level is distinctly below the detected magnetic noise generated by the test sample itself. In case of a scanning speed of 1 revolution/s about 12000 samples are recorded for each channel, corresponding to a spatial sampling of $55 \mu \mathrm{m}$.

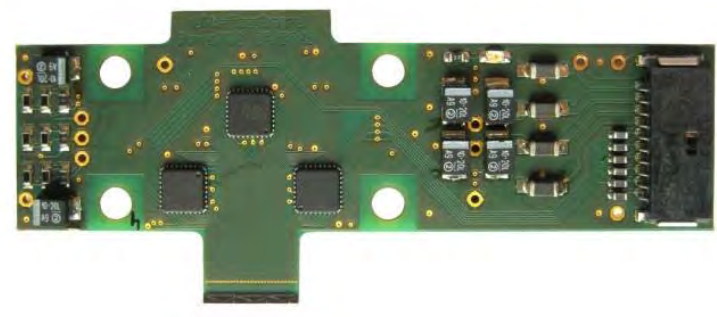

Fig. 4: Board including GMR-arrays and electronics. 


\section{Experimental results}

Fig. 5 shows some results of an inspected roller bearing (ø $215 \mathrm{~mm}$ ) where we introduced low energy EDM-notches (electrical discharge machining). The depth of the notches varies between $40 \mu \mathrm{m}$ up to $170 \mu \mathrm{m}$. Also the small notched were changed in orientation to inspect the sensitivity for different defect alignments. We used the adapted GMR gradiometer (Fig. 3) having dimensions of the sensing elements of $190 \mu \mathrm{m}$ followed of a $10 \mu \mathrm{m}$ distance to the next lateral element. having this size in mind and using a lift-off (sensor-to-sample distance) of about $100 \mu \mathrm{m}$ we also obtain a spatial resolution in the order of about $200 \mathrm{~mm}$.

Due to the gradiometric arrangement we observe in Fig. 5 (a) a relative flat response, which only significantly changes in presence of low energy EDM-notches. Otherwise when using a magnetometer the signatures also would contain background fields generated by the test specimen itself or magnetized components of the testing system. Using a vertical gradiometer also gives a response which is very similar to the magnetometer response. This is due to the fact that one GMR-layer is very close to the field generating defect, whereas the second GMR-layer positioned at the baseline with a larger distance to the defect sees only a fracture of the strayfield, but is

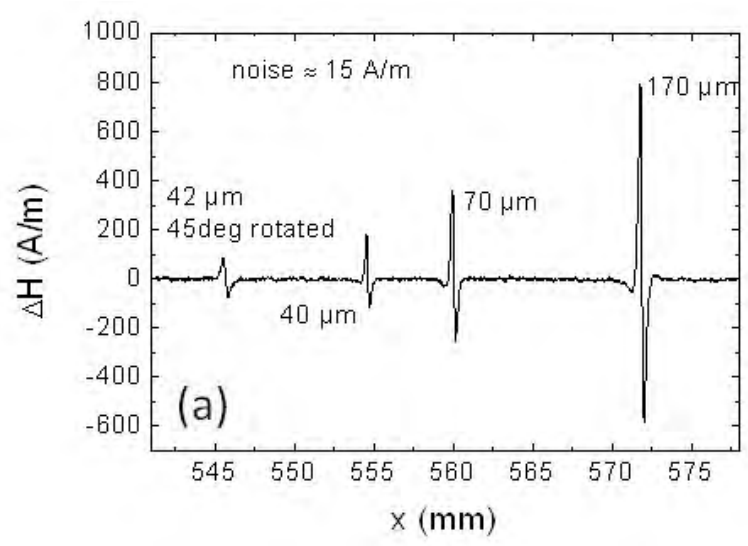

similar sensitive to homogeneous magnetic noise sources.

The deepest notch causes a peak-to-peak stray field signal strength of $1400 \mathrm{~A} / \mathrm{m}$ (somewhat more than $1 \mathrm{mT}$ ), whereas the smallest crack signal is about $180 \mathrm{~A} / \mathrm{m}$ caused by the $42 \mu \mathrm{m}$ deep crack which was rotated by $45^{\circ}$ (see Fig. 5 (b)). Here, the noise level is about $15 \mathrm{~A} / \mathrm{m}$, resulting in a SNR of about 12 . The $40 \mu \mathrm{m}$ deep crack and the $170 \mu \mathrm{m}$ deep crack show an peak-to-peak amplitude of about $300 \mathrm{~A} / \mathrm{m}$ and $1400 \mathrm{~A} / \mathrm{m}$, respectively, leading to an SNR of up to 90 . These high SNR-values can be explained by both the low distance between the surface and the sensor array of about $100 \mu \mathrm{m}$ and the small sensing area. Besides the SNR, this also leads to a high spatial resolution.

For further POD analysis (Probability of Detection) of the new inspection method we introduced a sufficiently high number of micronotches with depth varying from $10 \mu \mathrm{m}$ up to $300 \mu \mathrm{m}$ in some roller bearings (see Fig. 6). This analysis has to be done to quantitatively verify the testing reliability using adapted GMR sensors for MFL inspection. This is important to classify GMR inspection techniques within the deployed NDT methods like magnetic particle inspection and eddy current testing.

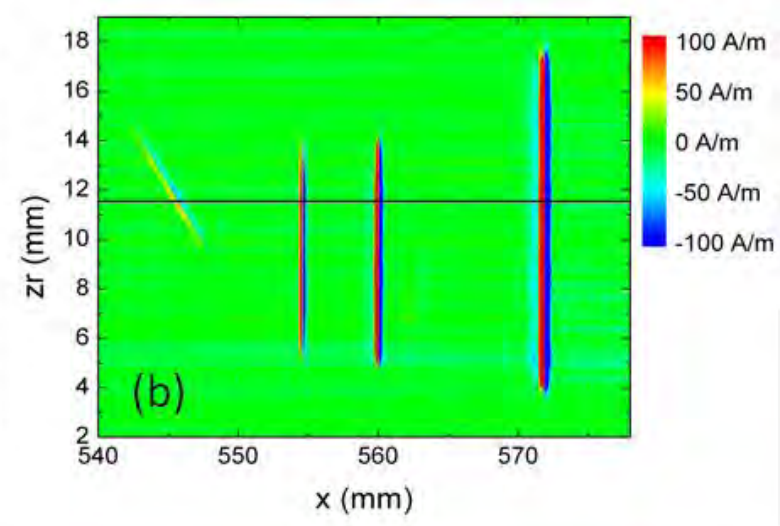

Fig. 5: (a) Difference of the vertical field component $\Delta H_{z}$ along the circumferential direction of the bearing at $z r=$ $11.5 \mathrm{~mm}$ (indicated by the solid line in (b), where four different EDM notches were introduced (The corresponding depths of the notches are indicated at each signal. (b) C-scan of the magnified area in false colour representation.
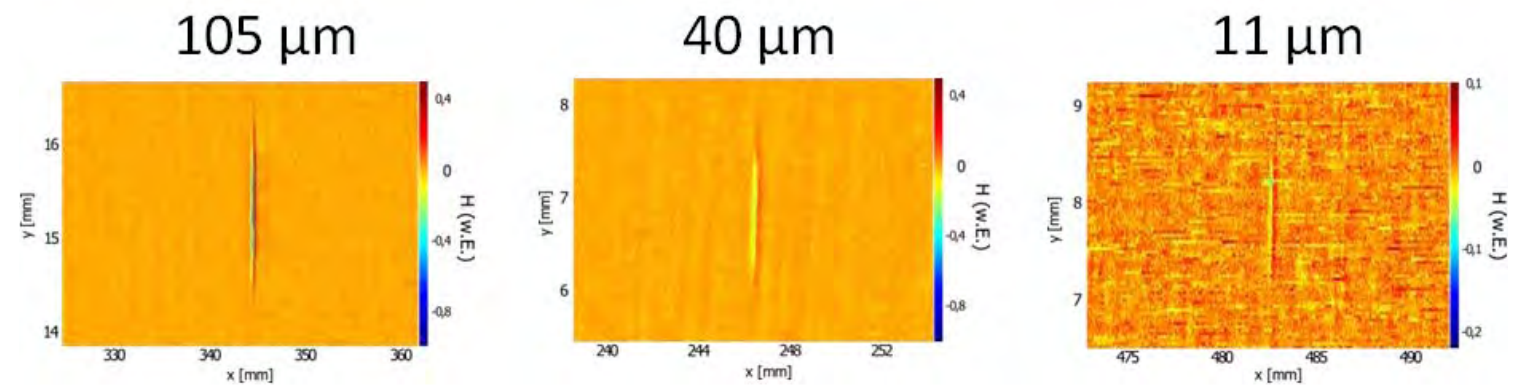

Fig. 6: magnetic stray field distribution above defects of a POD-roller bearing 105,40 , and 11 um deep notches. 

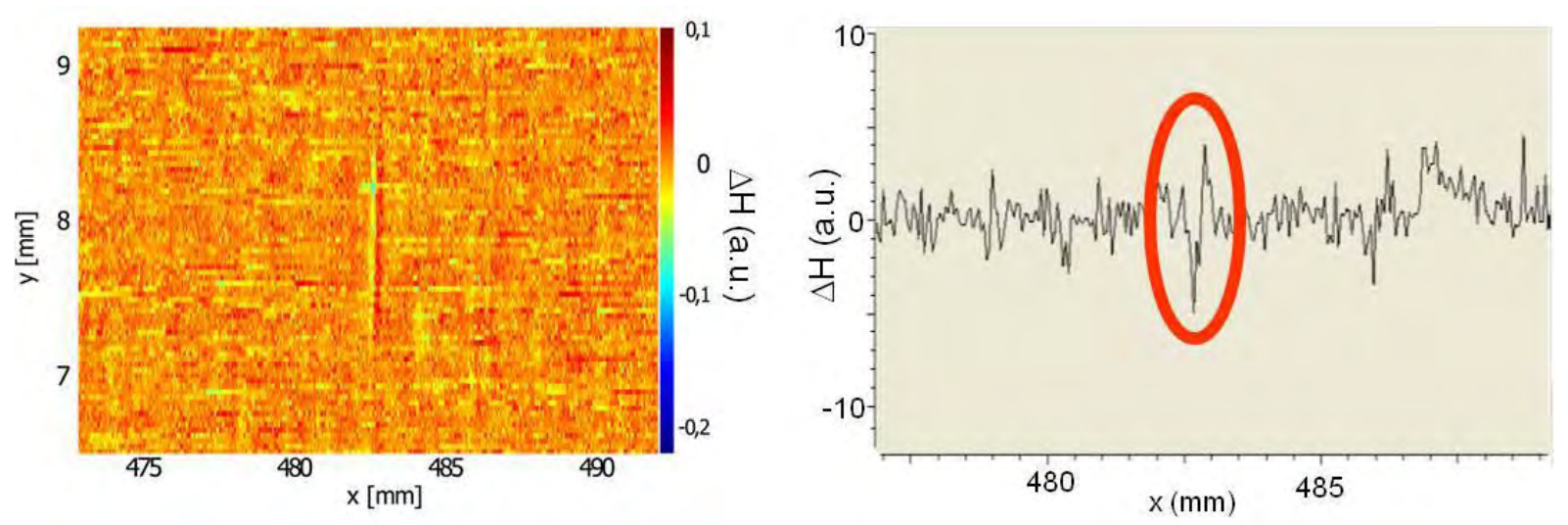

Fig. 7: $11 \mu m$ deep notch. Left: false rendering plot. Right: Line scan across the notch.

In Fig. 7 we show the MFL signal of the $11 \mu \mathrm{m}$ deep notch presented as a first result of our studies. The SNR is less than $6 \mathrm{~dB}$ for this small notch (see Fig. 7 right). For an automated detection this signal will be too low using simple algorithm for the detection. Nevertheless, the MFL could be resolved in the false rendering plot (see Fig. 7 left). A POD-analysis requires several hundred measurements to be performed for a statistical conclusion. This analysis is an ongoing working field at the NDT department at BAM.

\section{Reconstruction}

Reconstruction of the geometry of defects can be an important tool for non-destructive testing to evaluate the origin of defects and to control process parameters. Also, the spatial geometry can indicate further developments to overcome crack initiation.

Fig. 8 left depicts a scheme of the reconstruction procedure. In a first global step the magnetic field distribution inside the material under test is simulated. For this purpose we need the magnetic properties of the yoke and the material itself. Also, the geometries of all components have to be known. The knowledge of the distribution is then used for the reconstruction. Here, we are only interested in a small region - the so called "region of interest" (ROI). This region contains the defect. We estimate the defect depth using the data measured by the GMR sensor arrays. To prevent long computational time we adopt a priori knowledge like, e.g., sensor position and liftoff. During the reconstruction of the permeability the measured data is compared with actualized defect geometries. Here, it is important to implement the distances between sensor element and surface of the component under test. Also, the geometry of the sensor array like length of the GMR elements and tipping of the sensor are implemented as additional information. In doing so, it is possible to estimate the depth of the defects.
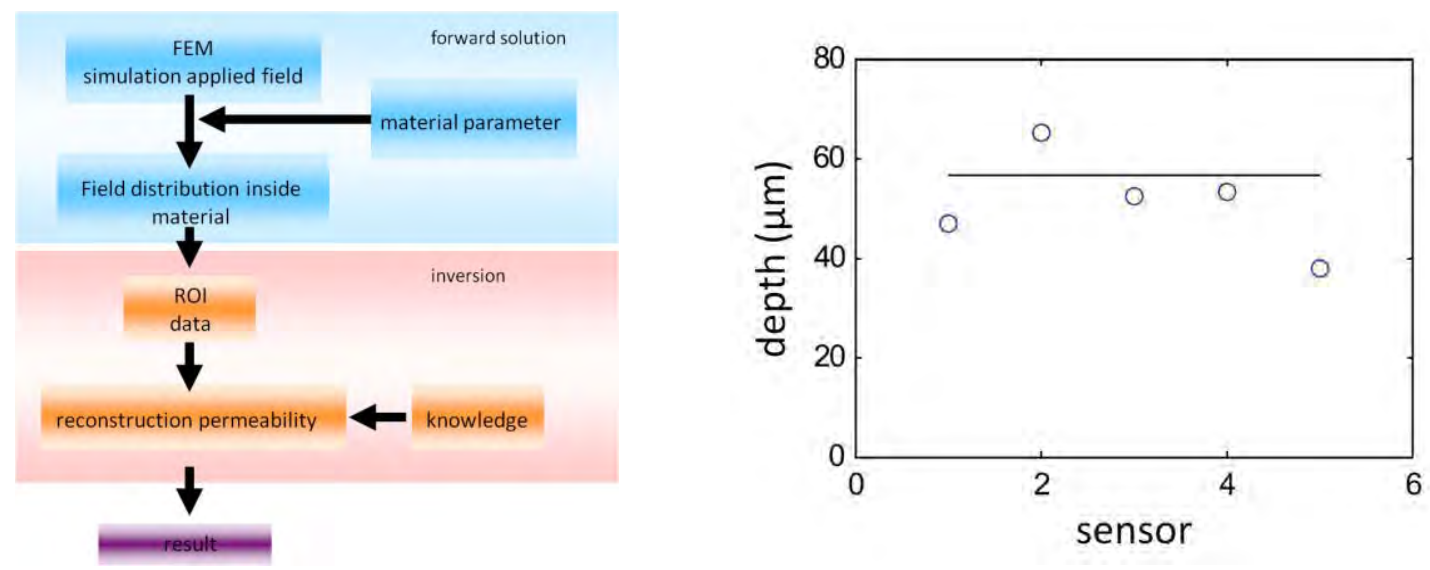

Fig. 8: Left: Scheme of the reconstruction steps. Right: reconstructed depth of a $57 \mu m$-deep notch as function of sensor position. The blue circles indicate the reconstruction result of sensor 1 - sensor 5 . 
In Fig. 8 right is a diagram of the reconstructed depth of $57 \mu \mathrm{m}$-deep notch for several sensor position shown. After 30 cycles the inversion converges. The computational time is in the order of a few seconds. At the center of the notch the depth can be reconstructed with small deviations. The defect has a length of $1000 \mu \mathrm{m}$. Using the sensor pitch of $200 \mu \mathrm{m}$ we have 3 sensor elements, which are above the defect with their full extension (sensor 2, 3, and 4). For the data observed by sensor elements near the edges of the notch (sensor 1 and 5) the reconstructed depth decreases. Here, the magnetic flux leakage decreases since the magnetic field inside the material will be guided around the notch instead of leaking from the material leading to smaller measured field values. Also, the profile of an EDM-notch must not be rectangular.

\section{Conclusion}

Electromagnetic testing based on small magneto resistive layers with high spatial resolution can be provided with relatively high cost effectiveness. This allows GMR-sensors to follow the miniaturization trend providing an adequate testing method for quality control of small components. GMR-technology thus has the potential to bridge the micro-gap between the mm-sized conventional induction coils for detecting macroscopic material defects and the scanning magnetic force microscopy for the detection of field distribution on the $\mathrm{nm}$-scale.

Detecting the magnetic field close to the surface offers the advantage to obtain more detailed information on the geometrical parameters of the defects. Using a few $\mu \mathrm{m}$ sized GMR gradiometer, where the sensing MR-layer is positioned close to the chip edge, we obtained distinct crack signatures with good spatial resolution.

The automation of flux leakage testing is an active research field. The use of adapted magnetic field sensors to detect stray field distributions of defects above the test specimen can be a promising feature for Nondestructive Testing.

\section{Acknowledgement}

This work was supported by the German Federal Ministry of Education and Research (grant no. 16SV3787). We also would like to thank our partners Astro- und Feinwerktechnik Adlershof $\mathrm{GmbH}$, Prüftechnik Linke und Rühe $\mathrm{GmbH}$ and SKF Germany. We further thank Uwe Loreit and Claudia Glenske from Sensitec $\mathrm{GmbH}$ for fruitful discussion.

\section{References}

[1] Baibich, M.; Broto, J.; Fert, A.; Nguyen van Dau, F.; Petroff, F.; Eitenne, P.; Cruezet, G.; Friederich, A.; Chazelas, J. Giant magnetoresistance of $(001) \mathrm{Fe} /(001) \mathrm{Cr}$ magnetic superlattices. Phys. Rev. Lett. 1988, 66, 24722475.

[2] Binasch, G.; Grünberg, G.; Saurenbach, F.; Zinn, $W$. Enhanced magnetoresistance in layered magnetic structures with antiferromagnetic interlayer exchange. Phys. Rev. B 1989, 57, 4828-4830.

[3] Daughton, J.M. GMR applications. J. Magn. Mater. 1999, 192, 334-342.

[4] Bakonyi, I.; Péter, L. Electrodeposited multilayer films with giant magnetoresistance (GMR): Progress and problems. Prog. Mater. Sci. 2010, 55, 107-245.

[5] Christides, C.; Panagiotopoulos, I.; Niarchos, D.; Jones, G. Fast magnetic field mapping of permanent magnets with GMR bridge and hallprobe sensors. Sens. Actuat. A: Phys. 2003, 106, 243-245.

[6] Kataoka, Y.; Murayama, S.; Wakiwaka, H.; Shinoura, O. Application of GMR line sensor to detect the magnetic flux distribution for nondestructive testing. Int. J. Appl. Electromagn. Mech. 2001/2002, 15, 47-52.

[7] Pelkner, M.; Neubauer, A.; Blome, M.; Reimund, V.; Thomas, H.-M.; Kreutzbruck, M. Flux leakage measurements for defect characterization using NDT adapted GMR sensors. In Studies in Applied Electromagnetics and Mechanics; IOP Press: Fairfax, VA, USA, 2011, pp. 217-224.

[8] Kreutzbruck, M.; Allweins, K.; Strackbein, C.; Bernau, H. Inverse algorithm for electromagnetic wire inspection based on GMR-Sensor arrays. Int. J. Appl. Electromagn. Mech. 2009, 30, 299-308.

[9] Dolabdjian, C.P.; Perez, L.; de Haan, V.O.; de Jong, P.A. Performance of magnetic pulsededdy-current system using high dynamic and high linearity improved giant magneto resistance magnetometer. IEEE Sens. J. 2006, 6, 15111517.

[10] Cacciola, M.; Megali, G.; Pallicanó, D.; Morabito, F.C. A GMR-ECT based embedded solution for applications on PCB inspections. Sens. Actuat. A: Phys. 2011, 167, 25-33.

[11] Dogaru, T.; Smith, S.T. Giant magnetoresistancebased eddy-current sensor. IEEE Trans. Magn. 2001, 37, 3831-3838.

[12] Kim, J.; Yang, G.; Udpa, L.; Udpa, S. Classification of pulsed eddy current GMR data on aircraft structures. NDT\&E Int. 2010, 43, 141144.

[13] M. Pelkner, A. Neubauer, V. Reimund and M. Kreutzbruck, "Routes for GMR-Sensor Design in Non-Destructive Testing", Sensors, 12 (2012), 12169-12183. 